\title{
An atlas on risk factors for type 2 diabetes: a wide-angled Mendelian randomisation study
}

\author{
Shuai Yuan $^{1}$ (D) Susanna C. Larsson ${ }^{1,2}$ (D) \\ Received: 27 April 2020 / Accepted: 10 July 2020 / Published online: 8 September 2020 \\ (C) The Author(s) 2020
}

\begin{abstract}
Aims/hypothesis The aim of this study was to use Mendelian randomisation (MR) to identify the causal risk factors for type 2 diabetes.

Methods We first conducted a review of meta-analyses and review articles to pinpoint possible risk factors for type 2 diabetes. Around 170 possible risk factors were identified of which 97 risk factors with available genetic instrumental variables were included in MR analyses. To reveal more risk factors that were not included in our MR analyses, we conducted a review of published MR studies of type 2 diabetes. For our MR analyses, we used summary-level data from the DIAbetes Genetics Replication And Meta-analysis consortium (74,124 type 2 diabetes cases and 824,006 controls of European ancestry). Potential causal associations were replicated using the FinnGen consortium (11,006 type 2 diabetes cases and 82,655 controls of European ancestry). The inverse-variance weighted method was used as the main analysis. Multivariable MR analysis was used to assess whether the observed associations with type 2 diabetes were mediated by BMI. We used the Benjamini-Hochberg method that controls false discovery rate for multiple testing.

Results We found evidence of causal associations between 34 exposures (19 risk factors and 15 protective factors) and type 2 diabetes. Insomnia was identified as a novel risk factor (OR 1.17 [95\% CI 1.11, 1.23]). The other 18 risk factors were depression, systolic BP, smoking initiation, lifetime smoking, coffee (caffeine) consumption, plasma isoleucine, valine and leucine, liver alanine aminotransferase, childhood and adulthood BMI, body fat percentage, visceral fat mass, resting heart rate, and four plasma fatty acids. The 15 exposures associated with a decreased risk of type 2 diabetes were plasma alanine, HDL- and total cholesterol, age at menarche, testosterone levels, sex hormone binding globulin levels (adjusted for BMI), birthweight, adulthood height, lean body mass (for women), four plasma fatty acids, circulating 25-hydroxyvitamin D and education years. Eight associations remained after adjustment for adulthood BMI. We additionally identified 21 suggestive risk factors $(p<0.05)$, such as alcohol consumption, breakfast skipping, daytime napping, short sleep, urinary sodium, and certain amino acids and inflammatory factors.

Conclusions/interpretation The present study verified several previously reported risk factors and identified novel potential risk factors for type 2 diabetes. Prevention strategies for type 2 diabetes should be considered from multiple perspectives on obesity, mental health, sleep quality, education level, birthweight and smoking.
\end{abstract}

Electronic supplementary material The online version of this article (https://doi.org/10.1007/s00125-020-05253-x) contains supplementary material, which is available to authorised users.

Susanna C. Larsson

susanna.larsson@surgsci.uu.se

1 Unit of Cardiovascular and Nutritional Epidemiology, Institute of Environmental Medicine, Karolinska Institutet, Nobelsväg 13, 17177 Stockholm, Sweden
2 Department of Surgical Sciences, Uppsala University, Dag Hammarskjölds Väg 14B, 75185 Uppsala, Sweden 


\section{Research in context}

\section{What is already known about this subject?}

- Observational studies have revealed a large number of possible risk factors for type 2 diabetes; however, it remains unclear whether these recognised associations are causal

\section{What is the key question?}

- Are identified risk factors for type 2 diabetes in observational studies causally associated with risk of type 2 diabetes?

\section{What are the new findings?}

- We found evidence of causal associations between 34 exposures (19 risk factors and 15 protective factors) and type 2 diabetes, and eight associations remained after adjustment for adulthood BMI

- Insomnia was identified as a novel risk factor for type 2 diabetes

- We additionally identified 21 suggestive risk factors, including several lifestyle factors and certain amino acids and inflammatory factors

How might this impact on clinical practice in the foreseeable future?

- Prevention strategies for type 2 diabetes should be considered from multiple perspectives on obesity, mental health, sleep quality, education level, birthweight and smoking

Keywords Mendelian randomisation $\cdot$ Prevention $\cdot$ Review $\cdot$ Risk factors $\cdot$ Type 2 diabetes

$\begin{array}{ll}\begin{array}{l}\text { Abbreviations } \\ \text { DIAGRAM }\end{array} & \begin{array}{l}\text { DIAbetes Genetics Replication And Meta- } \\ \text { analysis consortium }\end{array} \\ \text { GWAS } & \begin{array}{l}\text { Genome-wide association study } \\ \text { MR }\end{array} \\ \text { HRC } & \text { Hendelian randomisation } \\ \text { SHBG } & \text { Sex hormone binding globulin }\end{array}$

\section{Introduction}

Type 2 diabetes is a global public health issue, affecting 9 in 100 adults worldwide in 2015 according to the International Diabetes Federation $[1,2]$. The increasing prevalence of type 2 diabetes along with severe complications cause an immense disease and economic burden [1,3]. Therefore, it is important to better understand the aetiological basis of type 2 diabetes and establish prevention strategies.

Reviews of observational studies have revealed a large number of possible risk factors for type 2 diabetes covering health status, dietary and lifestyle factors, environmental factors, and different biomarkers [4-6]. However, whether the reported associations are causal remains unclear due to potential methodological limitations in the observational studies, such as confounding and reverse causality. For certain exposures with defined detrimental influences on human health, such as smoking and heavy alcohol drinking, it is unfeasible to determine their causal associations with type 2 diabetes in an experimental setting.

Utilising genetic variants as instrumental variables for an exposure, Mendelian randomisation (MR) analysis can strengthen the causal inference on an exposure-outcome association [7, 8]. The MR study design has two major strengths when compared with the traditional observational design (electronic supplementary material [ESM] Fig. 1). First, the result of an MR analysis is less likely to be driven by confounding because genetic variants are randomly allocated at conception and, therefore, one trait is generally unrelated to other traits. This resembles the random assignment of participants to experimental and control groups in an RCT [7-9]. Second, an MR analysis avoids reverse causality as alleles are fixed and cannot be modified by the onset and progression of a disease [7, 8].

Several previous MR studies have examined the associations of various exposures, such as nutritional, lifestyle, obesity-related, hormone-related and inflammatory factors and internal biomarkers, with type 2 diabetes (ESM Table 1). However, there has been no study systematically assessing the causal effects of possible risk factors on type 2 diabetes. Here, we conducted an MR investigation to determine the causal associations of a wide range of possible risk factors proposed by observational studies with type 2 diabetes risk. 


\section{Methods}

Study design overview The overview of study design is presented in Fig. 1. To identify possible risk factors for type 2 diabetes, we conducted a review of meta-analyses and review articles identified by a search in the PubMed database. In total, around 170 possible risk factors were pinpointed of which 97 risk factors with available genetic instrumental variables were included in the present MR study. In addition, we conducted a review of published MR studies of type 2 diabetes to reveal more risk factors that were not included in the present study. This MR study was approved by the Swedish Ethical Review Authority.

Data source for type 2 diabetes Summary-level data for type 2 diabetes were available in the DIAbetes Genetics Replication And Meta-analysis (DIAGRAM) consortium, which includes 32 studies with a total of 898,130 individuals of European descent (74,124 cases and 824,006 controls) [10]. Participants had a mean age of around 55 years and 51.8\% were men. The Haplotype Reference Consortium (HRC) reference panel was used in the imputation stage and adjustments were made for population structure (e.g. through principal components), relatedness and study-specific covariates. Considering that BMI may mediate the associations between certain exposures and type 2 diabetes, we used the summarylevel estimates from the genome-wide association analysis without BMI adjustment.

Data from the FinnGen consortium (11,006 type 2 diabetes cases and 82,655 controls) were used in the replication stage (https://www.finngen.fi/fi). Detailed methods (e.g. participating biobanks/cohorts, data collection, genotyping and data analysis) are presented in its webpage.
Data source for adulthood BMI Summary-level data for BMI were obtained from a recent genome-wide association study (GWAS) of 806,834 individuals of European ancestry (including Genetic Investigation of ANthropometric Traits [GIANT] consortium and UK Biobank) [11]. The HRC reference panel was used in the imputation stage and adjustments were made for age, sex and principal components of ancestry. Results were validated in an independent dataset including 7721 UK individuals.

Instrumental variable selection SNPs associated with each exposure at the genome-wide significance threshold $(p<5 \times$ $10^{-8}$ ) were proposed as instrumental variables for 97 exposures from corresponding GWASs. We excluded SNPs in linkage disequilibrium $\left(R^{2} \geq 0.01\right)$. For each trait, all instrumental variables were harmonised so that the effect alleles reflected the allele associated with increased probability, prevalence or levels of the exposure. For SNPs not available in the type 2 diabetes dataset, proxy SNPs were searched in the dataset of the National Cancer Institute, Division of Cancer Epidemiology \& Genetics (https://ldlink.nci.nih.gov/) by setting $R^{2} \geq 0.8$. We replaced missing SNPs with proxy SNPs for traits with $\leq 10$ missing SNPs. Detailed information for data sources and SNPs used is shown in ESM Table 2. Definitions for exposures are presented in ESM Table 3.

A review of previous MR studies Many associations were tested in previous studies with an MR design. In order to reveal more causal risk factors for type 2 diabetes, we conducted a review of previous MR studies of possible risk factors for type 2 diabetes. Detailed methods are shown in the ESM Methods. In total, 238 papers were screened, and 40 individual studies were included. Publication data, number of SNPs used as

Review of
possible risk factors
We conducted a
review of meta-
analyses and review
articles on risk
factors for type 2
diabetes
In total, around 170
possible risk factors
were pinpointed

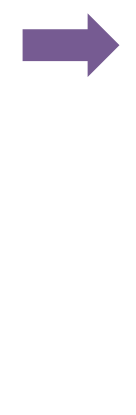

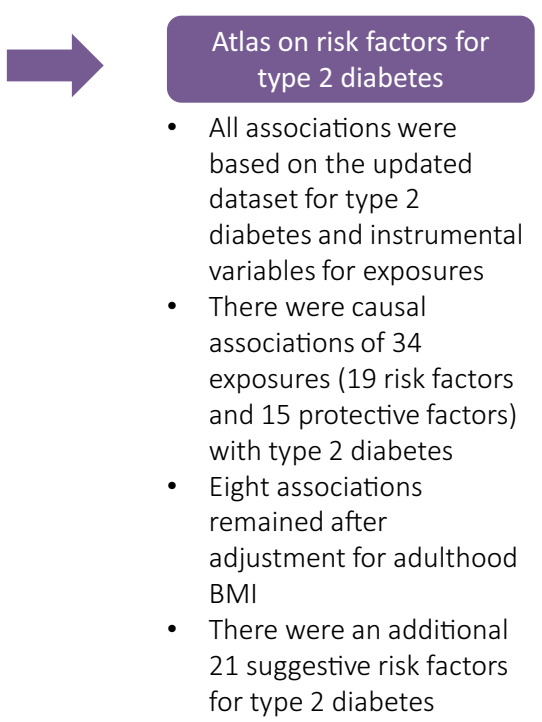

Fig. 1 Overview of study design 
instrumental variables, outcome source information and effect estimates (95\% CIs) were extracted.

Statistical analysis The $F$-statistic was estimated to examine the strength of the genetic instrument for each exposure and an $F$-statistic above 10 was considered a sufficiently strong instrument. The inverse-variance weighted method was used as the main analysis [12]. For exposures instrumented by $\geq 3$ SNPs, the overall estimates were calculated using the randomeffects inverse-variance weighted method. For exposures with only 1 or 2 SNPs available as instrumental variables, the fixed-effects inverse-variance weighted method was used. The inverse-variance weighted method can provide the most precise estimates but is sensitive to invalid instrumental variables and pleiotropic effects [12]. We additionally performed several sensitivity analyses based on the weighted median [13] and MR-Egger regression [14] methods to test the consistency of the associations under the different assumptions and to detect possible pleiotropy. Assuming that more than $50 \%$ of the weight in the analysis comes from valid instrumental variables, the weighted median method can provide an accurate estimate of the causal effect of the exposure on outcome [13]. MR-Egger regression can identify and correct for directional pleiotropy, but the estimation is of low precision [14]. We used $I^{2}$ to represent the heterogeneity among used SNPs [15] and defined the horizontal pleiotropy according to the $p$ value for the intercept in the MR-Egger model [16]. Considering the partial overlap of participants included in the GWASs of certain exposures and the DIAGRAM consortium, we replicated the associations with $p<0.05$ in the inverse-variance weighted model using an independent GWAS datasetFinnGen consortium. To evaluate whether the exposures that were associated with type 2 diabetes at $p \leq 0.05$ in the univariable analysis have a direct effect on type 2 diabetes not mediated by BMI, we performed a multivariable random-effects inverse-variance weighted analysis [17] adjusting for adulthood BMI. Since we aimed at assessing the effect of exposure of interest only, we adopted the multivariable MR method testing for mediation by BMI, rather than allowing for an independent effect of BMI as well as mediation by BMI simultaneously.

ORs and $95 \%$ CIs of type 2 diabetes were scaled to the unit reported in ESM Table 2. We conducted a general power analysis using a webtool [18] for MR analysis (ESM Table 4). All analyses were performed in Stata/SE 15.0 using the mrrobust [19] and in $\mathrm{R} 3.6 .0$ using the MendelianRandomization package [20]. All estimates were reported with two-tailed $p$ values. We used the BenjaminiHochberg method that controls the false discovery rate (FDR) for multiple testing [21]. Results from this analysis are presented in ESM Table 5. Associations with a Benjamini-Hochberg adjusted $p$ value $<0.05$ were regarded as significant. Suggestive causal associations were defined based on a comprehensive consideration of $p$ values for the estimates in inverse-variance weighted method and/or weighted median method and the consistency across analyses $(p<0.05$ in inverse-variance weighted or weighted median models and the direction of the association remained consistent in two models).

\section{Results}

Summary of results of this MR investigation and review of previous MR studies Among 97 exposures examined in this MR investigation (Table 1 and ESM Table 6), 29 were nominally associated with type 2 diabetes $(p<0.05)$ of which 20 were associated with type 2 diabetes after BenjaminiHochberg adjustment for multiple comparisons (ESM Tables 5 and 6). Combining those 20 significant associations with 14 significant associations (Table 2) identified by our review of previous MR studies (ESM Table 1), we found evidence of causal associations of 34 exposures with risk of type 2 diabetes. In detail, an increased risk of type 2 diabetes was observed with 19 exposures: depression, insomnia, systolic BP, smoking initiation, lifetime smoking, coffee (caffeine) consumption, plasma isoleucine, valine and leucine, liver alanine aminotransferase, childhood and adulthood BMI, body fat percentage, visceral fat mass, resting heart rate, and four plasma fatty acids. A decreased risk of type 2 diabetes was observed with 15 exposures: plasma alanine, HDL- and total cholesterol, age at menarche, testosterone levels, sex hormone binding globulin (SHBG) levels (adjusted for $\mathrm{BMI}$ ), birthweight, adulthood height, lean body mass (for women), four plasma fatty acids, circulating 25 hydroxyvitamin D and education years. Eight of the associations remained in the multivariable MR analysis adjusted for adulthood BMI (Fig. 2). There were a further 21 suggestive causal factors for type 2 diabetes, including alcohol consumption, breakfast skipping, daytime napping, short sleep, urinary sodium, and certain amino acids and inflammatory factors. Details of the univariable and multivariable MR analyses and the review of previous MR studies are presented below.

Univariable MR analysis The associations of the 97 exposures examined in this MR study with type 2 diabetes are presented in ESM Table 6. After Benjamini-Hochberg adjustment, we found evidence of causal associations of 20 exposures with type 2 diabetes. Among these exposures, 11 were associated with an increased risk of type 2 diabetes: systolic BP, lifetime smoking, insomnia, plasma isoleucine, valine and leucine, liver alanine aminotransferase, childhood and adulthood BMI, body fat percentage and visceral fat mass. Eight exposures were inversely associated with type 2 diabetes: plasma alanine, HDL- and total cholesterol, age at menarche, testosterone levels, SHBG levels (adjusted for BMI), birthweight 
Table 1 Associations between risk factors and type 2 diabetes in the present study using the latest DIAGRAM consortium (74,124 cases and 824,006 controls) and comparison with previous MR studies

Previous MR study conducted (yes/no)
Published association with diabetes PubMed identifier of (if available)

OR $(95 \% \mathrm{CI})$
Association with diabetes in the present MR study OR $(95 \% \mathrm{CI})$

\begin{tabular}{|c|c|c|c|c|}
\hline \multicolumn{5}{|l|}{ Somatic health status } \\
\hline Asthma & No & NA & & $1.01(0.94,1.10)$ \\
\hline Atopic dermatitis & No & NA & & $1.05(0.98,1.11)$ \\
\hline Dupuytren's disease & No & NA & & $1.01(0.99,1.03)$ \\
\hline Giant cell arteritis & No & NA & & $1.07(1.00,1.14)$ \\
\hline Hyperthyroidism & No & NA & & $1.00(0.95,1.04)$ \\
\hline Hypothyroidism & No & NA & & $0.99(0.95,1.04)$ \\
\hline Microalbuminuria & No & NA & & $1.26(0.86,1.85)$ \\
\hline $\begin{array}{c}\text { Microvascular } \\
\text { dysfunction }\end{array}$ & No & NA & & $1.03(0.96,1.10)$ \\
\hline Osteoarthritis & No & NA & & $0.94(0.83,1.07)$ \\
\hline Periodontitis & No & NA & & $1.03(1.00,1.06)$ \\
\hline $\begin{array}{l}\text { Polycystic ovary } \\
\text { syndrome }\end{array}$ & No & NA & & $0.96(0.92,1.01)$ \\
\hline Rheumatoid arthritis & No & NA & & $0.98(0.92,1.04)$ \\
\hline Systolic BP & Yes & $1.02(1.01,1.03)$ & $27702834[51]$ & $1.39(1.26,1.54)$ \\
\hline Telomere length & Yes & $1.00(0.84,1.20)$ & $28241208[52]$ & $1.02(0.96,1.08)$ \\
\hline \multicolumn{5}{|l|}{ Mental health status } \\
\hline Anorexia nervosa & No & NA & & $0.92(0.84,1.02)$ \\
\hline $\begin{array}{l}\text { Lifetime anxiety } \\
\text { disorder }\end{array}$ & No & NA & & $1.05(0.94,1.18)$ \\
\hline $\begin{array}{l}\text { Post-traumatic stress } \\
\text { disorder }\end{array}$ & No & NA & & $0.96(0.87,1.05)$ \\
\hline Schizophrenia & No & NA & & $1.00(0.97,1.04)$ \\
\hline \multicolumn{5}{|c|}{ Nutritional factor and internal biomarker } \\
\hline $\begin{array}{l}\beta \text {-carotenoid (precursor } \\
\text { to vitamin A) }\end{array}$ & Yes & $0.98(0.91,1.04)$ & $19662379[53]$ & $0.96(0.89,1.05)$ \\
\hline Retinol (vitamin A) & No & NA & & $1.15(0.85,1.56)$ \\
\hline Vitamin $\mathrm{B}_{6}$ & No & NA & & $1.00(0.99,1.00)$ \\
\hline Folate (vitamin $\mathrm{B}_{9}$ ) & No & NA & & $0.88(0.78,0.99)$ \\
\hline Vitamin $B_{12}$ & Yes & $0.96(0.71,1.30)$ & $29982347[54]$ & $0.99(0.95,1.04)$ \\
\hline Vitamin C & No & NA & & $1.00(0.99,1.01)$ \\
\hline Vitamin E & No & NA & & $1.21(0.76,1.93)$ \\
\hline Copper & No & NA & & $1.03(0.98,1.07)$ \\
\hline Iron & Yes & $0.89(0.81,0.98)$ & $30759836[55]$ & $1.06(0.99,1.13)$ \\
\hline Magnesium & Yes & $1.55(0.26,9.25)$ & $30759836[55]$ & $1.08(0.92,1.26)$ \\
\hline Potassium (urinary) & No & NA & & $0.71(0.32,1.61)$ \\
\hline Sodium (urinary) & No & NA & & $2.69(1.14,6.34)$ \\
\hline Selenium & Yes & $1.18(0.97,1.43)$ & $29788239[56]$ & $1.05(1.01,1.10)$ \\
\hline Zinc & Yes & $1.01(0.92,1.12)$ & $30759836[55]$ & $1.00(0.96,1.04)$ \\
\hline $\begin{array}{l}\text { Thyroid-stimulating } \\
\text { hormone }\end{array}$ & Yes & $0.91(0.78,1.07)$ & 28323940 [57] & $0.96(0.87,1.06)$ \\
\hline Free thyroxine & Yes & $1.10(0.94,1.30)$ & 28323940 [57] & $0.94(0.88,1.01)$ \\
\hline HDL-cholesterol & Yes & $0.83(0.76,0.90)$ & $27487401[58]$ & $0.78(0.67,0.91)$ \\
\hline LDL-cholesterol & Yes & $0.86(0.80,0.93)$ & $27487401[58]$ & $0.91(0.83,1.00)$ \\
\hline Total cholesterol & No & NA & & $0.87(0.79,0.96)$ \\
\hline Total triacylglycerol & Yes & $1.01(0.91,1.11)$ & $27487401[58]$ & $1.04(0.83,1.29)$ \\
\hline Lipoprotein(a) & No & NA & & $1.02(0.99,1.03)$ \\
\hline
\end{tabular}


Table 1 (continued)

Previous MR study conducted (yes/no)
Published association with diabetes PubMed identifier of (if available) OR $(95 \% \mathrm{CI})$
Association with diabetes in the present MR study OR $(95 \% \mathrm{CI})$

$1.02(1.00,1.03)$

$1.00(0.98,1.01)$

$1.00(0.99,1.01)$

$1.01(0.87,1.18)$

$1.06(0.83,1.36)$

$1.02(1.00,1.05)$

$1.10(0.87,1.38)$

$1.09(0.95,1.25)$

$1.26(1.16,1.37)$

$1.28(1.10,1.49)$

$1.23(1.08,1.39)$

$0.51(0.45,0.58)$

$1.15(1.03,1.29)$

$0.86(0.76,0.98)$

$0.98(0.82,1.17)$

$1.31(0.90,1.91)$

$1.02(0.86,1.20)$

$1.04(0.98,1.11)$

$1.13(1.01,1.27)$

$0.98(0.94,1.01)$

$0.99(0.98,1.00)$

$0.97(0.94,1.00)$

$1.05(0.93,1.18)$

$31024619[67] \quad 1.00(0.96,1.04)$

$0.99(0.96,1.01)$

$0.98(0.92,1.04)$

$1.01(0.97,1.06)$

$0.96(0.90,1.03)$

$1.08(0.80,1.45)$

$27845333[68] \quad 1.59(1.09,2.32)$

$1.17(1.09,1.25)$

$1.72(0.85,3.46)$

$31852999[26] \quad 1.61(1.36,1.91)$

$1.77(0.73,4.24)$

$0.83(0.62,1.12)$

$1.14(0.92,1.41)$

$0.79(0.47,1.34)$

$1.05(0.99,1.12)$

$1.17(1.11,1.23)$

$1.00(0.94,1.05)$

$1.01(0.98,1.05)$ 
Table 1 (continued)

\begin{tabular}{|c|c|c|c|c|}
\hline & $\begin{array}{l}\text { Previous MR study } \\
\text { conducted (yes/no) }\end{array}$ & $\begin{array}{l}\text { Published association with diabetes } \\
\text { (if available) } \\
\text { OR }(95 \% \text { CI })\end{array}$ & $\begin{array}{l}\text { PubMed identifier of } \\
\text { published MR }\end{array}$ & $\begin{array}{l}\text { Association with diabetes in the } \\
\text { present MR study } \\
\text { OR }(95 \% \mathrm{CI})\end{array}$ \\
\hline $\begin{array}{l}\text { Moderate to vigorous } \\
\text { physical activity }\end{array}$ & No & NA & & $0.69(0.22,2.14)$ \\
\hline $\begin{array}{l}\text { Strenuous sports or } \\
\text { other exercises }\end{array}$ & No & NA & & $0.77(0.42,1.72)$ \\
\hline $\begin{array}{l}\text { Vigorous physical } \\
\text { activity }\end{array}$ & No & NA & & $0.90(0.78,1.04)$ \\
\hline Accelerometry & No & NA & & $0.98(0.95,1.02)$ \\
\hline \multicolumn{5}{|l|}{ Sex-related factor } \\
\hline Age at menarche & Yes & $0.83(0.78,0.88)$ & $31614369[70]$ & $0.84(0.80,0.88)$ \\
\hline Age at menopause & No & NA & & $0.99(0.96,1.01)$ \\
\hline Testosterone levels & Yes & $1.07(0.80,1.43)$ & $\operatorname{medR} \chi$ iv [71] & $0.72(0.59,0.89)$ \\
\hline SHBG & Yes & $0.83(0.76,0.91)$ & $26050255[72]$ & $0.55(0.47,0.64)$ \\
\hline Oestradiol levels & No & NA & & $0.99(0.87,1.13)$ \\
\hline \multicolumn{5}{|l|}{ Obesity-related factor } \\
\hline Birthweight & No & $2.79(1.90,4.20)$ & 31539074 [73] & $0.79(0.67,0.92)$ \\
\hline Childhood BMI & No & $1.83(1.46,2.30)$ & 29483184 [74] & $1.87(1.44,2.44)$ \\
\hline Adulthood BMI & No & $1.31(1.11,1.53)$ & $31821322[75]$ & $1.89(1.73,2.07)$ \\
\hline Adulthood height & No & NA & & $0.95(0.92,0.98)$ \\
\hline Body fat percentage & No & NA & & $2.07(1.79,2.39)$ \\
\hline Visceral fat mass & No & $\begin{array}{l}2.50(1.98,3.14) \text { for men } 7.34 \\
\quad(4.48,12.0) \text { for women }\end{array}$ & $31501611[76]$ & $2.63(2.14,3.23)$ \\
\hline Circulating adiponectin & No & NA & & $0.82(0.63,1.07)$ \\
\hline Leptin levels & No & NA & & $1.13(0.24,5.37)$ \\
\hline
\end{tabular}

NA, not available

Table 2 Established associations of 14 risk factors with type 2 diabetes in a review of previous MR studies using the latest DIAGRAM consortium $(74,124$ cases and 824,006 controls)

\begin{tabular}{|c|c|c|c|c|c|c|}
\hline Risk factor & PMID & Year & SNPs & OR & $95 \% \mathrm{CI}$ & Unit \\
\hline Resting heart rate & 31648709 [77] & 2019 & Genetic score & 1.12 & $1.11,1.12$ & 10 beats/min \\
\hline$\alpha$-Linolenic acid & 31690987 [78] & 2019 & 1 & 0.93 & $0.90,0.96$ & $\mathrm{SD}$ \\
\hline Eicosapentaenoic acid & 31690987 [78] & 2019 & 2 & 1.08 & $1.03,1.12$ & $\mathrm{SD}$ \\
\hline Docosapentaenoic acid & 31690987 [78] & 2019 & 2 & 1.04 & $1.02,1.07$ & $\mathrm{SD}$ \\
\hline Linoleic acid & 31690987 [78] & 2019 & 3 & 0.96 & $0.94,0.98$ & $\mathrm{SD}$ \\
\hline Arachidonic acid & 31690987 [78] & 2019 & 2 & 1.03 & $1.02,1.05$ & $\mathrm{SD}$ \\
\hline Palmitoleic acid & 31690987 [78] & 2019 & 4 & 0.86 & $0.81,0.91$ & $\mathrm{SD}$ \\
\hline Oleic acid & 31690987 [78] & 2019 & 1 & 0.87 & $0.81,0.93$ & $\mathrm{SD}$ \\
\hline Stearic acid & 31690987 [78] & 2019 & 3 & 1.09 & $1.09,1.15$ & $\mathrm{SD}$ \\
\hline 25-hydroxyvitamin D & 31548248 [79] & 2019 & 7 & 0.94 & $0.88,0.99$ & $\mathrm{SD}$ \\
\hline Smoking initiation & $31852999[26]$ & 2019 & 377 & 1.28 & $1.20,1.37$ & $\mathrm{NA}$ \\
\hline Fat free mass (women) & $30798333[80]$ & 2019 & 311 & 0.91 & $0.84,0.99$ & $\mathrm{SD}$ \\
\hline Depression $^{\text {a }}$ & $32270255[48]$ & 2020 & 89 & 1.26 & $1.10,1.43$ & Prevalence \\
\hline Education years ${ }^{\text {a }}$ & medR $\chi i v ~[49]$ & 2020 & 1263 & 0.53 & $0.50,0.57$ & $\mathrm{SD}$ \\
\hline
\end{tabular}

${ }^{a}$ Results for depression and education years were not from review and from our previous studies. The study on depression has been published, however the study on education years has not yet been officially published. The doi for education paper: https://doi.org/10.1101/2020.02.01.20020008

NA, not available 


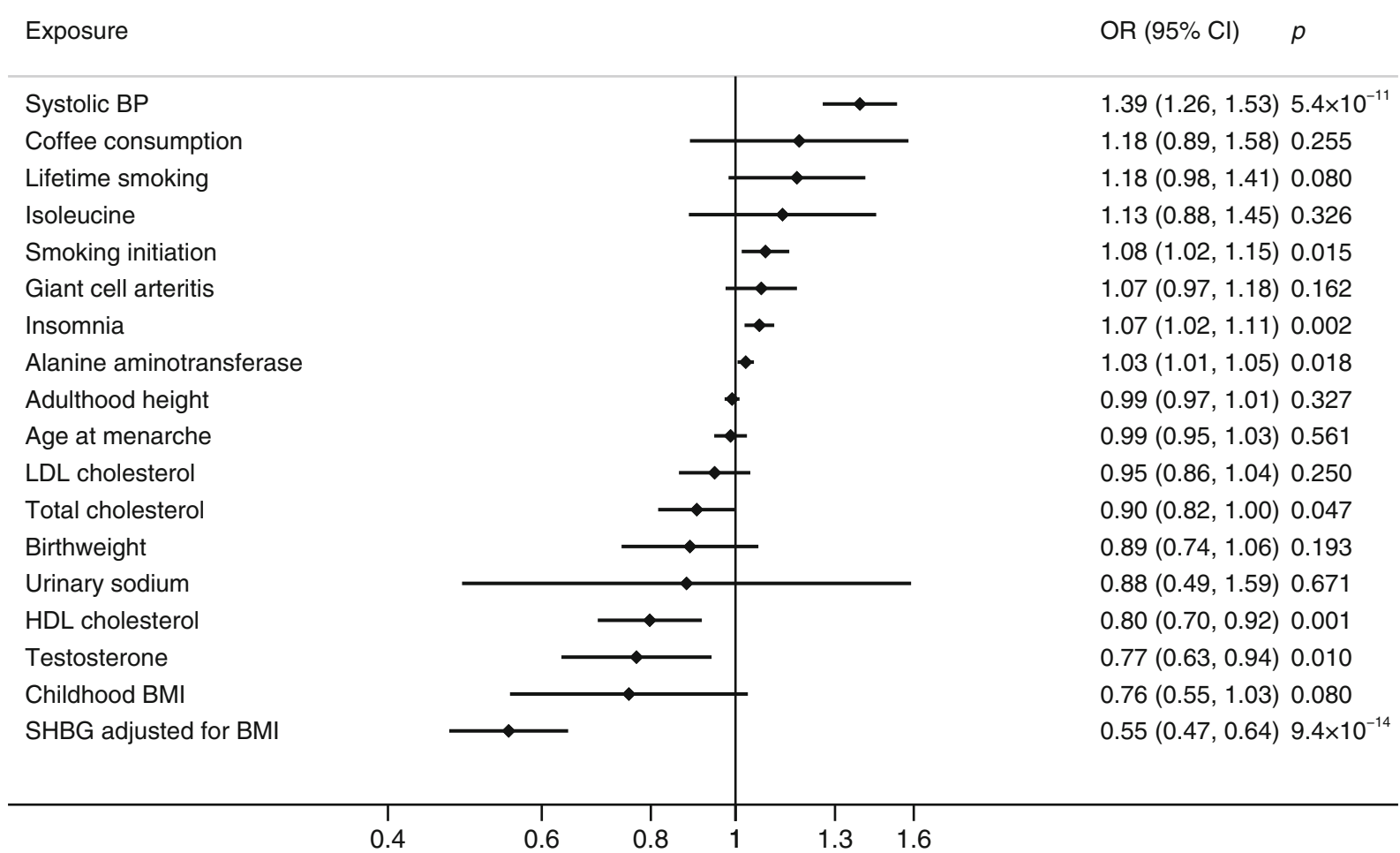

OR $(95 \% \mathrm{Cl})$ of type 2 diabetes after adjusting for adulthood BMI

Fig. 2 Associations of risk factors identified for type 2 diabetes in the univariable MR analysis with type 2 diabetes after adjustment for adulthood BMI. Traits that highly correlated with adulthood BMI, such as

and adulthood height. The associations for HDL-cholesterol and height with type 2 diabetes were inconsistent in the weighted median analysis. There was possible pleiotropy in the association of testosterone and SHBG levels (adjusted for BMI) with type 2 diabetes. In addition, we found suggestive evidence of associations between 21 exposures and type 2 diabetes: giant cell arteritis, atopic dermatitis, lifetime anxiety disorder, serum iron and selenium, urinary sodium, thyroid-stimulating hormone, LDLcholesterol, total triacylglycerol, fetuin-A levels, homocysteine, phenylalanine, tyrosine, IL-1 receptor antagonist, IL-6 receptor subunit $\alpha$, TNF, alcohol consumption, breakfast skipping, daytime napping, short sleep duration and morningness.

For alcohol consumption, the result of the weighted median analysis showed a positive association between alcohol consumption and type 2 diabetes (OR 1.46 [95\% CI 1.16, 1.83]) (ESM Table 6). After adjustment for pleiotropy in the MR-Egger regression analysis, the OR of type 2 diabetes was 2.27 (95\% CI 1.30, 3.93) per SD increase of log-transformed drinks/week (ESM Table 6). In addition, rs1229984 in the $A D H 1 B$ gene, which is robustly associated with alcohol consumption and explains the majority of the variance of alcohol consumption, was positively associated with type 2 diabetes (OR 1.57 [95\% CI 1.20, 2.07]) (ESM Fig. 2). Coffee consumption, mainly driven by SNPs in the CYP1A1/2 and $A H R$ genes which are also associated with higher caffeine intake but lower blood caffeine metabolites, was positively visceral fat mass and body fat percentage, were not included. SHBG, sex hormone binding globulin

associated with type 2 diabetes in the inverse-variance weighted and weighted median models (ESM Table 6).

Replication results in FinnGen for associations with $p<0.05$ in the inverse-variance model are presented in ESM Table 7. More than half of the associations reached the $p$ value $<0.1$ and all associations were in the same direction as in the analyses based on data from the DIAGRAM consortium.

Multivariable MR analysis Results of the multivariable MR analysis are shown in Fig. 2. Eight risk factors remained associated with type 2 diabetes at $p<0.05$ after adjustment for adulthood BMI. Specifically, systolic BP, smoking, insomnia and alanine aminotransferase levels were positively associated with type 2 diabetes, whereas testosterone, SHBG, and HDLand total cholesterol levels were inversely associated with type 2 diabetes after BMI adjustment.

\section{Discussion}

In the present MR investigation and complementary review of previous MR studies, we found evidence of causal associations of 34 exposures with type 2 diabetes. Most of the associations were identified in previous MR studies and replicated in the present MR study using a larger dataset for type 2 diabetes and/or more instrumental variables for the exposures. 
Insomnia was identified as a novel causal risk factor for type 2 diabetes. Eight risk factors were related to type 2 diabetes after adjustment for BMI, suggesting that those exposures affect the risk of type 2 diabetes independently of BMI. In addition, we identified 21 suggestive risk factors for type 2 diabetes, such as alcohol consumption, breakfast skipping, daytime napping, short sleep, and certain amino acids and inflammatory factors.

Comparison with previous MR studies Previous MR studies based on data from the largest GWAS meta-analysis of type 2 diabetes from the DIAGRAM consortium revealed associations of genetically predicted resting heart rate, plasma phospholipid levels of eight fatty acids, serum 25-hydroxyvitamin D levels, smoking initiation, lean body mass (for women), depression and education levels with type 2 diabetes. In addition, there were associations for isoleucine, leucine, valine, phylloquinone (vitamin $\mathrm{K}_{1}$ ), IL-6 receptor, IL-18, HDL- and LDL-cholesterol, alanine aminotransferase, aspartate aminotransferase, alkaline phosphatase, bilirubin levels, age at menarche, SHBG, visceral fat mass, birthweight, childhood and adulthood BMI, WHR adjusted for BMI and IGFbinding protein-3 levels with type 2 diabetes. Results for serum homocysteine and C-reactive protein levels were inconsistent.

The present MR investigation confirmed most results of previous MR studies. However, some associations did not persist when using updated data (more instrumental variables) for the exposure or outcome (more cases and controls), including associations for phylloquinone (vitamin $\mathrm{K}_{1}$ ), IL-18, liver aspartate aminotransferase, alkaline phosphatase and bilirubin. Findings were inconsistent regarding the effects of Creactive protein $[22,23]$ and plasma homocysteine levels $[24,25]$ on type 2 diabetes in previous studies. In the present MR study, there was limited evidence supporting causal associations of C-reactive protein. However, plasma homocysteine levels showed suggestive association with type 2 diabetes. Smoking initiation has also been identified as a strong risk factor for type 2 diabetes [26]. Here, we used an instrument that predicts lifetime smoking exposure and verified the causal detrimental effect of smoking on type 2 diabetes risk.

Novel potential risk factors Insomnia with objective short sleep duration has been associated with an increased risk of type 2 diabetes in observational studies [27, 28]. The present MR study found strong and suggestive evidence of a causal association of insomnia and short sleep duration, respectively, with increased risk of type 2 diabetes. We did not detect an effect of sleep duration on type 2 diabetes in MR analysis assuming a linear trend. This finding is consistent with those of observational studies which have shown a U-shaped relationship between sleep duration and diabetes risk [29], although an association with long sleep duration might reflect reverse causality. In addition, observational studies have associated daytime napping with an increased risk of type 2 diabetes [30], which is supported by our MR findings. Short sleep and poor sleep quality have been shown to be associated with less healthy eating and irregular meal patterns, including breakfast skipping [31], which was related to an increased risk of type 2 diabetes in this MR study and in previous observational studies [32].

Data on the association between giant cell arteritis and type 2 diabetes are scarce. A Danish cohort study found that patients with giant cell arteritis $(n=1682)$ had a markedly increased risk of new-onset diabetes compared with the general population [33], which is in line with our finding. Even though the CIs became broader after adjustment for BMI, the OR estimate was unchanged in our study. Moreover, the opposite effects of BMI on giant cell arteritis [34] and type 2 diabetes [35] in observational studies indicates that giant cell arteritis may be a causal risk factor for diabetes independently of BMI status.

The present study provided evidence that alcohol consumption may be a risk factor for type 2 diabetes. In particular, the alcohol-raising allele of the $A D H 1 B$ variant was strongly associated with an increased risk of type 2 diabetes. Some observational studies have indicated that light or moderate alcohol consumption is associated with a decreased risk of type 2 diabetes [36]. However, in an updated meta-analysis with 1,902,605 participants (including 125,926 individuals with type 2 diabetes), the inverse association between moderate drinking and type 2 diabetes was confined to certain subgroups [36]. Furthermore, the observed inverse association may be overestimated due to the inclusion of less healthy former drinkers in the reference group [37].

We found suggestive evidence that genetically predicted higher coffee and caffeine intake is associated with increased risk of type 2 diabetes, though the association with coffee consumption did not persist after adjustment for BMI. At first glance, these results appear contradictory to those of observational studies, which have consistently shown an inverse association between coffee consumption and type 2 diabetes incidence [38]. Nevertheless, the alleles of the variants in the CYP1A1/A2 and $A H R$ genes that predict higher caffeine consumption are related to faster caffeine metabolism and significantly lower blood levels of caffeine and higher paraxanthine-to-caffeine ratio [39]. It can thus be speculated that higher circulating levels of caffeine may be protective against type 2 diabetes.

Urinary sodium levels reflect dietary sodium to some extent. Observational studies have found that both higher urinary sodium excretion and dietary sodium intake were associated with higher risk of type 2 diabetes [40, 41], which is supported by our MR findings. In addition, consistent with the protective effects of high educational attainment on type 2 diabetes in observational studies [42], the present study confirmed that genetically predicted higher education level was associated with a lower diabetes risk. After adjustment for BMI, the association attenuated but persisted, which demonstrates that education may influence type 2 diabetes risk 
through BMI as well as other pathways, such as lowering psychological risk and smoking rate and levels.

Atopic dermatitis, anxiety, fetuin-A levels, phenylalanine, daytime napping and morningness were identified as novel possible causal risk factors. However, observational findings were inconclusive on atopic dermatitis [43] and anxiety [44, 45] and scarce on morningness. The established roles of fetuin-A levels [46], phenylalanine [47] and daytime napping [30] in the present study were in line with most of the observational studies. Considering limited SNPs and small variance explained by SNPs used for these traits, associations for these exposures with type 2 diabetes should be interpreted with caution and need further verification in studies in causal nature.

BMI and other risk factors In this study, seven of 15 exposuretype 2 diabetes associations attenuated but remained significant after adjustment for adulthood BMI, along with BMIindependent effects observed for depression [48] and education [49], which implies that adiposity is a strong risk factor for type 2 diabetes but also that controlling for BMI cannot fully prevent type 2 diabetes. Considering the large effect of obesity on type 2 diabetes [35] and an increasing global burden of obesity [50], an emphasis on weight control via healthier food choices and physical activity is needed. Simultaneously, other strategies focusing on other risk factors also merit attention, such as improving mental health status and sleep quality in developed areas, improving educational level and birthweight in developing areas and advocating antismoking actions worldwide.

Strengths and limitations This is the first study that has comprehensively assessed the causal associations between a large number of exposures and type 2 diabetes using the latest summary-level data for type 2 diabetes. The use of the MR design strengthened the causal inference on the exposurediabetes associations due to diminished residual confounding and reverse causality. We also conducted a review to identify the main possible risk factors for type 2 diabetes. Most causal associations were replicated in an independent consortium. In addition, several sensitivity analyses were performed to test the consistency of results and reveal and correct for possible pleiotropy. Multivariable MR analysis uncovered several obesity-independent risk factors for type 2 diabetes, which provides new thinking for type 2 diabetes prevention. We used a combined design of original MR analysis and review and, therefore, extended the study scope to some extent in both risk factor detection and association revelation. Even though we pinpointed a large number of possible risk factors by conducting a review of meta-analyses and review articles on risk factors for type 2 diabetes, some risk factors may have been missed due to a scoped review design and the lack of genetic instruments for certain exposures. Another limitation is that we might have overlooked weak associations, especially for traits with small variance explained by SNPs used. However, the power would be $\geq 70 \%$ if the SNPs explained $1 \%$ variance of a phenotype for a risk factor with an $\mathrm{OR} \geq 1.1$ or $\leq 0.9$. Another limitation is that the instrumental strength may have been low in some of the multivariable MR analyses. Instrumental variables selection was based on a mixed population for some traits, which might introduce population bias. However, the majority of participants in these corresponding GWASs were of European descent and their analyses adjusted for population principal components. Additionally, many exposures for type 2 diabetes identified in observational data cannot be assessed in the MR design due to no available instrumental variables until now.

Conclusions The present MR study verified several previously established risk factors and identified novel potential risk factors for type 2 diabetes using the latest summary-level data. Findings should inform public health policies for the primary prevention of type 2 diabetes. Prevention strategies should be constructed from multiple perspectives, such as lowering obesity and smoking rates and levels, and improving mental health, sleep quality, educational level and birthweight.

Acknowledgements Summary-level data for genetic associations with type 2 diabetes were obtained from the DIAGRAM consortium. The authors thank all investigators for sharing these data.

Data availability Genetic instruments can be obtained from the individual referenced papers. Summary-level genetic data for type 2 diabetes can be downloaded at the website: https://www.diagram-consortium.org/. The datasets analysed in this study are publicly available summary statistics.

Funding Information Open access funding provided by Karolinska Institute. Funding for this study was received from the Swedish Research Council (Vetenskapsrådet; Grant number 2019-00977) and the Swedish Research Council for Health, Working Life and Welfare (Forte; Grant number 2018-00123).

Authors' relationships and activities The authors declare that there are no relationships or activities that might bias, or be perceived to bias, their work.

Contribution statement SY and SCL designed the study and contributed to the interpretation of the results and critical revision of the manuscript for important intellectual content. SY conducted the systematic review, performed the statistical analyses and drafted the manuscript. All authors read and approved the final version of the manuscript. SY is responsible for the integrity of the work as a whole.

Open Access This article is licensed under a Creative Commons Attribution 4.0 International License, which permits use, sharing, adaptation, distribution and reproduction in any medium or format, as long as you give appropriate credit to the original author(s) and the source, provide a link to the Creative Commons licence, and indicate if changes were made. The images or other third party material in this article are included in the article's Creative Commons licence, unless indicated otherwise in a credit line to the material. If material is not included in the article's Creative Commons licence and your intended use is not 
permitted by statutory regulation or exceeds the permitted use, you will need to obtain permission directly from the copyright holder. To view a copy of this licence, visit http://creativecommons.org/licenses/by/4.0/.

\section{References}

1. Zheng Y, Ley SH, Hu FB (2018) Global aetiology and epidemiology of type 2 diabetes mellitus and its complications. Nat Rev Endocrinol 14(2):88-98. https://doi.org/10.1038/nrendo.2017.151

2. Federation ID (2020) IDF Diabetes atlas 9th edn 2019. Available from https://diabetesatlas.org/en/ Accessed 20 Jan 2020

3. Naqshbandi M, Harris SB, Esler JG, Antwi-Nsiah F (2008) Global complication rates of type 2 diabetes in indigenous peoples: a comprehensive review. Diabetes Res Clin Pract 82(1):1-17. https://doi.org/10.1016/j.diabres.2008.07.017

4. Bellou V, Belbasis L, Tzoulaki I, Evangelou E (2018) Risk factors for type 2 diabetes mellitus: an exposure-wide umbrella review of meta-analyses. PLoS One 13(3):e0194127. https://doi.org/10.1371/ journal.pone. 0194127

5. Dendup T, Feng X, Clingan S, Astell-Burt T (2018) Environmental risk factors for developing type 2 diabetes mellitus: a systematic review. Int J Environ Res Public Health 15(1):78. https://doi.org/ 10.3390/ijerph15010078

6. Sattar N, Wannamethee SG, Forouhi NG (2008) Novel biochemical risk factors for type 2 diabetes: pathogenic insights or prediction possibilities? Diabetologia 51(6):926-940. https://doi.org/10.1007/ s00125-008-0954-7

7. Davey Smith G, Ebrahim S (2005) What can mendelian randomisation tell us about modifiable behavioural and environmental exposures? BMJ 330(7499):1076-1079. https://doi.org/10. 1136/bmj.330.7499.1076

8. Burgess S, Thompson SG (2015) Mendelian randomization: methods for using genetic variants in causal estimation. Chapman and Hall/CRC Press, London, UK

9. Haycock PC, Burgess S, Wade KH, Bowden J, Relton C, Davey Smith G (2016) Best (but oft-forgotten) practices: the design, analysis, and interpretation of Mendelian randomization studies. Am J Clin Nutr 103(4):965-978. https://doi.org/10.3945/ajen.115. 118216

10. Mahajan A, Taliun D, Thurner M et al (2018) Fine-mapping type 2 diabetes loci to single-variant resolution using high-density imputation and islet-specific epigenome maps. Nat Genet 50(11):15051513. https://doi.org/10.1038/s41588-018-0241-6

11. Pulit SL, Stoneman C, Morris AP et al (2019) Meta-analysis of genome-wide association studies for body fat distribution in 694 649 individuals of European ancestry. Hum Mol Genet 28(1):166174. https://doi.org/10.1093/hmg/ddy327

12. Burgess S, Bowden J, Fall T, Ingelsson E, Thompson SG (2017) Sensitivity analyses for robust causal inference from Mendelian randomization analyses with multiple genetic variants. Epidemiology 28(1):30-42. https://doi.org/10.1097/ede. 0000000000000559

13. Bowden J, Davey Smith G, Haycock PC, Burgess S (2016) Consistent estimation in Mendelian randomization with some invalid instruments using a weighted median estimator. Genet Epidemiol 40(4):304-314. https://doi.org/10.1002/gepi.21965

14. Burgess S, Thompson SG (2017) Interpreting findings from Mendelian randomization using the MR-Egger method. Eur J Epidemiol 32(5):377-389. https://doi.org/10.1007/s10654-0170255-x

15. Greco MF, Minelli C, Sheehan NA, Thompson JR (2015) Detecting pleiotropy in Mendelian randomisation studies with summary data and a continuous outcome. Stat Med 34(21):29262940. https://doi.org/10.1002/sim.6522

16. Bowden J, Davey Smith G, Burgess S (2015) Mendelian randomization with invalid instruments: effect estimation and bias detection through Egger regression. Int J Epidemiol 44(2):512-525. https://doi.org/10.1093/ije/dyv080

17. Burgess S, Thompson SG (2015) Multivariable Mendelian randomization: the use of pleiotropic genetic variants to estimate causal effects. Am J Epidemiol 181(4):251-260. https://doi.org/10.1093/ aje/kwu283

18. Brion MJ, Shakhbazov K, Visscher PM (2013) Calculating statistical power in Mendelian randomization studies. Int $\mathrm{J}$ Epidemiol 42(5):1497-1501. https://doi.org/10.1093/ije/dyt179

19. Spiller W, Davies NM, Palmer TM (2019) Software application profile: mrrobust - a tool for performing two-sample summary Mendelian randomization analyses. Int J Epidemiol. https://doi. org/10.1093/ije/dyy195

20. Yavorska OO, Burgess S (2017) MendelianRandomization: an R package for performing Mendelian randomization analyses using summarized data. Int J Epidemiol 46(6):1734-1739. https://doi.org/ 10.1093/ije/dyx034

21. Benjamini Y, Hochberg Y (1995) Controlling the false discovery rate: a practical and powerful approach to multiple testing. J R Stat Soc Ser B Stat Methodol 57(1):11. https://doi.org/10.1111/j.25176161.1995.tb02031.x

22. Noordam R, Oudt CH, Bos MM, Smit RAJ, van Heemst D (2018) High-sensitivity C-reactive protein, low-grade systemic inflammation and type 2 diabetes mellitus: a two-sample Mendelian randomization study. Nutr Metab Cardiovasc Dis 28(8):795-802. https:// doi.org/10.1016/j.numecd.2018.03.008

23. Cheng L, Zhuang H, Yang S, Jiang H, Wang S, Zhang J (2018) Exposing the causal effect of C-reactive protein on the risk of type 2 diabetes mellitus: a Mendelian randomization study. Front Genet 9: 657. https://doi.org/10.3389/fgene.2018.00657

24. Kumar J, Ingelsson E, Lind L, Fall T (2015) No evidence of a causal relationship between plasma homocysteine and type 2 diabetes: a Mendelian randomization study. Front Cardiovasc Med 2:11. https://doi.org/10.3389/fcvm.2015.00011

25. Huang T, Ren J, Huang J, Li D (2013) Association of homocysteine with type 2 diabetes: a meta-analysis implementing Mendelian randomization approach. BMC Genomics 14:867. https://doi.org/ $10.1186 / 1471-2164-14-867$

26. Yuan S, Larsson SC (2019) A causal relationship between cigarette smoking and type 2 diabetes mellitus: a Mendelian randomization study. Sci Rep 9(1):19342. https://doi.org/10.1038/s41598-01956014-9

27. Vgontzas AN, Liao D, Pejovic S, Calhoun S, Karataraki M, Bixler EO (2009) Insomnia with objective short sleep duration is associated with type 2 diabetes: a population-based study. Diabetes Care 32(11):1980-1985. https://doi.org/10.2337/dc09-0284

28. Hein M, Lanquart JP, Loas G, Hubain P, Linkowski P (2018) Prevalence and risk factors of type 2 diabetes in insomnia sufferers: a study on 1311 individuals referred for sleep examinations. Sleep Med 46:37-45. https://doi.org/10.1016/j.sleep.2018.02.006

29. Shan Z, Ma H, Xie M et al (2015) Sleep duration and risk of type 2 diabetes: a meta-analysis of prospective studies. Diabetes Care 38(3):529-537. https://doi.org/10.2337/dc14-2073

30. Chen GC, Liu MM, Chen LH et al (2018) Daytime napping and risk of type 2 diabetes: a meta-analysis of prospective studies. Sleep Breath 22(3):815-824. https://doi.org/10.1007/s11325-017-1528-Z

31. Theorell-Haglow J, Lemming EW, Michaelsson K, Elmstahl S, Lind L, Lindberg E (2020) Sleep duration is associated with healthy diet scores and meal patterns: results from the population-based EpiHealth study. J Clin Sleep Med 16(1):9-18. https://doi.org/10. $5664 /$ jcsm. 8112 
32. Baliunas DO, Taylor BJ, Irving H et al (2009) Alcohol as a risk factor for type 2 diabetes: a systematic review and meta-analysis. Diabetes Care 32(11):2123-2132. https://doi.org/10.2337/dc090227

33. Faurschou M, Ahlstrom MG, Lindhardsen J, Obel N, Baslund B (2017) Risk of diabetes mellitus among patients diagnosed with giant cell arteritis or granulomatosis with polyangiitis: comparison with the general population. J Rheumatol 44(1):78-83. https://doi. org/10.3899/jrheum.160797

34. Jakobsson K, Jacobsson L, Warrington K et al (2015) Body mass index and the risk of giant cell arteritis: results from a prospective study. Rheumatology (Oxford) 54(3):433-440. https://doi.org/10. 1093/rheumatology/keu331

35. Lyall DM, Celis-Morales C, Ward J et al (2017) Association of body mass index with cardiometabolic disease in the UK Biobank: a Mendelian randomization study. JAMA Cardiol 2(8): 882-889. https://doi.org/10.1001/jamacardio.2016.5804

36. Li XH, Yu FF, Zhou YH, He J (2016) Association between alcohol consumption and the risk of incident type 2 diabetes: a systematic review and dose-response meta-analysis. Am J Clin Nutr 103(3): 818-829. https://doi.org/10.3945/ajcn.115.114389

37. Knott C, Bell S, Britton A (2015) Alcohol consumption and the risk of type 2 diabetes: a systematic review and dose-response metaanalysis of more than 1.9 million individuals from 38 observational studies. Diabetes Care 38(9):1804-1812. https://doi.org/10.2337/ dc15-0710

38. Carlstrom M, Larsson SC (2018) Coffee consumption and reduced risk of developing type 2 diabetes: a systematic review with metaanalysis. Nutr Rev 76(6):395-417. https://doi.org/10.1093/nutrit/ nuy014

39. Cornelis MC, Munafo MR (2018) Mendelian randomization studies of coffee and caffeine consumption. Nutrients 10(10):1343. https://doi.org/10.3390/nu10101343

40. Hu G, Jousilahti P, Peltonen M, Lindstrom J, Tuomilehto J (2005) Urinary sodium and potassium excretion and the risk of type 2 diabetes: a prospective study in Finland. Diabetologia 48(8): 1477-1483. https://doi.org/10.1007/s00125-005-1824-1

41. Provenzano LF, Stark S, Steenkiste A, Piraino B, Sevick MA (2014) Dietary sodium intake in type 2 diabetes. Clin Diabetes 32(3):106-112. https://doi.org/10.2337/diaclin.32.3.106

42. Kivimaki M, Batty GD, Pentti J et al (2020) Association between socioeconomic status and the development of mental and physical health conditions in adulthood: a multi-cohort study. Lancet Public Health. https://doi.org/10.1016/s2468-2667(19)30248-8

43. Thyssen JP, Halling-Overgaard AS, Andersen YMF, Gislason G, Skov L, Egeberg A (2018) The association with cardiovascular disease and type 2 diabetes in adults with atopic dermatitis: a systematic review and meta-analysis. Br J Dermatol 178(6):12721279. https://doi.org/10.1111/bjd.16215

44. Deschenes SS, Burns RJ, Graham E, Schmitz N (2016) Prediabetes, depressive and anxiety symptoms, and risk of type 2 diabetes: a community-based cohort study. J Psychosom Res 89:85-90. https://doi.org/10.1016/j.jpsychores.2016.08.011

45. Edwards LE, Mezuk B (2012) Anxiety and risk of type 2 diabetes: evidence from the Baltimore Epidemiologic Catchment Area Study. J Psychosom Res 73(6):418-423. https://doi.org/10.1016/j. jpsychores.2012.09.018

46. Roshanzamir F, Miraghajani M, Rouhani MH, Mansourian M, Ghiasvand R, Safavi SM (2018) The association between circulating fetuin-A levels and type 2 diabetes mellitus risk: systematic review and meta-analysis of observational studies. J Endocrinol Investig 41(1):33-47. https://doi.org/10.1007/s40618-017-0697-8

47. Merino J, Leong A, Liu CT et al (2018) Metabolomics insights into early type 2 diabetes pathogenesis and detection in individuals with normal fasting glucose. Diabetologia 61(6):1315-1324. https://doi. org/10.1007/s00125-018-4599-x
48. Tang B, Yuan S, Xiong Y, He Q, Larsson SC (2020) Major depressive disorder and cardiometabolic diseases: a bidirectional Mendelian randomisation study. Diabetologia. https://doi.org/10. 1007/s00125-020-05131-6

49. Yuan S, Xiong Y, Michaelsson M, Michaelsson K, Larsson SC (2020) Health related effects of education levels: a Mendelian randomization study. medR Xiv. https://doi.org/10.1101/2020.02. 01.20020008

50. Bluher M (2019) Obesity: global epidemiology and pathogenesis. Nat Rev Endocrinol 15(5):288-298. https://doi.org/10.1038/ s41574-019-0176-8

51. Aikens RC, Zhao W, Saleheen D et al (2017) Systolic blood pressure and risk of type 2 diabetes: a Mendelian randomization study. Diabetes 66(2):543-550. https://doi.org/10.2337/db16-0868

52. Telomeres Mendelian Randomization Collaboration, Haycock PC, Burgess S et al (2017) Association between telomere length and risk of cancer and non-neoplastic diseases: a Mendelian randomization study. JAMA Oncol 3(5):636-651. https://doi.org/10.1001/ jamaoncol.2016.5945

53. Perry JR, Ferrucci L, Bandinelli S et al (2009) Circulating $\beta$ carotene levels and type 2 diabetes-cause or effect? Diabetologia. 52(10):2117-2121. https://doi.org/10.1007/s00125-009-1475-8

54. Moen GH, Qvigstad E, Birkeland KI, Evans DM, Sommer C (2018) Are serum concentrations of vitamin B-12 causally related to cardiometabolic risk factors and disease? A Mendelian randomization study. Am J Clin Nutr 108(2):398-404. https://doi.org/10. 1093/ajen/nqy101

55. Cheng WW, Zhu Q, Zhang HY (2019) Mineral nutrition and the risk of chronic diseases: a Mendelian randomization study. Nutrients 11(2):378. https://doi.org/10.3390/nu11020378

56. Yarmolinsky J, Bonilla C, Haycock PC et al (2018) Circulating selenium and prostate cancer risk: a Mendelian randomization analysis. J Natl Cancer Inst 110(9):1035-1038. https://doi.org/10.1093/ jnci/djy081

57. Bos MM, Smit RAJ, Trompet S, van Heemst D, Noordam R (2017) Thyroid signaling, insulin resistance, and 2 diabetes mellitus: a Mendelian randomization study. J Clin Endocrinol Metab 102(6): 1960-1970. https://doi.org/10.1210/jc.2016-2816

58. White J, Swerdlow DI, Preiss D et al (2016) Association of lipid fractions with risks for coronary artery disease and diabetes. JAMA Cardiol 1(6):692-699. https://doi.org/10.1001/jamacardio.2016. 1884

59. De Silva NMG, Borges MC, Hingorani AD et al (2019) Liver function and risk of type 2 diabetes: bidirectional Mendelian randomization study. Diabetes 68(8):1681-1691. https://doi.org/ $10.2337 / \mathrm{db} 18-1048$

60. Keenan T, Zhao W, Rasheed A et al (2016) Causal assessment of serum urate levels in cardiometabolic diseases through a Mendelian randomization study. J Am Coll Cardiol 67(4):407-416. https://doi. org/10.1016/j.jacc.2015.10.086

61. Abbasi A (2015) Mendelian randomization studies of biomarkers and type 2 diabetes. Endocr Connect 4(4):249-260. https://doi.org/ 10.1530/EC-15-0087

62. Kröger J, Meidtner K, Stefan N et al (2018) Circulating fetuin-a and risk of type 2 diabetes: a Mendelian randomization analysis. Diabetes 67(6):1200-1205. https://doi.org/10.2337/db17-1268

63. Abbasi A, Deetman PE, Corpeleijn E et al (2015) Bilirubin as a potential causal factor in type 2 diabetes risk: a Mendelian randomization study. Diabetes 64(4):1459-1469. https://doi.org/10.2337/ db14-0228

64. Lotta LA, Scott RA, Sharp SJ et al (2016) Genetic predisposition to an impaired metabolism of the branched-chain amino acids and risk of type 2 diabetes: a Mendelian randomisation analysis. PLoS Med 13(11):e1002179. https://doi.org/10.1371/journal.pmed.1002179

65. Interleukin 1 Genetics Consortium (2015) Cardiometabolic effects of genetic upregulation of the interleukin 1 receptor antagonist: a 
Mendelian randomisation analysis. Lancet Diabetes Endocrinol 3(4):243-253. https://doi.org/10.1016/S2213-8587(15)00034-0

66. Interleukin-6 Receptor Mendelian Randomisation Analysis (IL6R MR) Consortium, Swerdlow DI, Holmes MV et al (2012) The interleukin-6 receptor as a target for prevention of coronary heart disease: a mendelian randomisation analysis. Lancet 379(9822): 1214-1224. https://doi.org/10.1016/S0140-6736(12)60110-X

67. Zhuang H, Han J, Cheng L, Liu SL (2019) A positive causal influence of IL-18 levels on the risk of T2DM: a Mendelian randomization study. Front Genet 10:295. https://doi.org/10.3389/fgene.2019. 00295

68. Kwok MK, Leung GM, Schooling CM (2016) Habitual coffee consumption and risk of type 2 diabetes, ischemic heart disease, depression and Alzheimer's disease: a Mendelian randomization study. Sci Rep 6:36500. https://doi.org/10.1038/srep36500

69. Wang J, Kwok MK, Au Yeung SL et al (2019) Sleep duration and risk of diabetes: observational and Mendelian randomization studies. Prev Med 119:24-30. https://doi.org/10.1016/j.ypmed.2018. 11.019

70. Cao M, Cui B (2020) Negative effects of age at menarche on risk of cardiometabolic diseases in adulthood: a Mendelian randomization study. J Clin Endocrinol Metab 105(2):dgz071. https://doi.org/10. 1210/clinem/dgz071

71. Mohammadi-Shemirani P, Chong M, Pigeyre M, Morton RW, Gerstein HC, Pare G (2019) Clinical benefits and adverse effects of genetically-elevated free testosterone levels: a Mendelian randomization analysis. medR $\chi$ iv. https://doi.org/10.1101/ 19005132

72. Wang Q, Kangas AJ, Soininen P et al (2015) Sex hormone-binding globulin associations with circulating lipids and metabolites and the risk for type 2 diabetes: observational and causal effect estimates. Int J Epidemiol 44(2):623-637. https://doi.org/10.1093/ije/dyv093

73. BIRTH-GENE (BIG) Study Working Group, Huang T, Wang T et al (2019) Association of birth weight with type 2 diabetes and glycemic traits: a Mendelian randomization study. JAMA Netw
Open 2(9):e1910915. https://doi.org/10.1001/jamanetworkopen. 2019.10915

74. Geng T, Smith CE, Li C, Huang T (2018) Childhood BMI and adult type 2 diabetes, coronary artery diseases, chronic kidney disease, and cardiometabolic traits: a Mendelian randomization analysis. Diabetes Care 41(5):1089-1096. https://doi.org/10.2337/dc172141

75. Wainberg M, Mahajan A, Kundaje A et al (2019) Homogeneity in the association of body mass index with type 2 diabetes across the UK Biobank: a Mendelian randomization study. PLoS Med 16(12): e1002982. https://doi.org/10.1371/journal.pmed.1002982

76. Karlsson T, Rask-Andersen M, Pan G et al (2019) Contribution of genetics to visceral adiposity and its relation to cardiovascular and metabolic disease. Nat Med 25(9):1390-1395. https://doi.org/10. 1038/s41591-019-0563-7

77. Guo Y, Chung W, Zhu Z et al (2019) Genome-wide assessment for resting heart rate and shared genetics with cardiometabolic traits and type 2 diabetes. J Am Coll Cardiol 74(17):2162-2174. https://doi.org/10.1016/j.jacc.2019.08.1055

78. Yuan S, Larsson SC (2020) Association of genetic variants related to plasma fatty acids with type 2 diabetes mellitus and glycaemic traits: a Mendelian randomisation study. Diabetologia 63(1):116123. https://doi.org/10.1007/s00125-019-05019-0

79. Yuan S, Jiang X, Michaëlsson K, Larsson SC (2019) Genetic prediction of serum 25-hydroxyvitamin d, calcium, and parathyroid hormone levels in relation to development of type 2 diabetes: a Mendelian randomization study. Diabetes Care 42(12):21972203. https://doi.org/10.2337/dc19-1247

80. Yeung CHC, Au Yeung SL, Fong SSM, Schooling CM (2019) Lean mass, grip strength and risk of type 2 diabetes: a bidirectional Mendelian randomisation study. Diabetologia 62(5): 789-799. https://doi.org/10.1007/s00125-019-4826-0

Publisher's note Springer Nature remains neutral with regard to jurisdictional claims in published maps and institutional affiliations. 